\title{
Effect of different operating modes and biomass concentrations on the recovery of recombinant hepatitis $B$ core antigen from thermal-treated unclarified Escherichia coli feedstock
}

\begin{abstract}
Expanded bed adsorption chromatography (EBAC) is a single pass operation that has been used as primary capture step in various protein purifications. The most common problem in EBAC is often associated with successful formation of a stable fluidized bed during the absorption stage, which is critically dependent on parameters such as liquid velocity, bed height, particle (adsorbent) size and density as well as design of column and type of flow distributor. In this study, residence time distribution (RTD) test using acetone as non-binding tracer acetone was performed to evaluate liquid dispersion characteristics of the EBAC system. A high $\mathrm{B}(\mathrm{o})$ number was obtained indicating the liquid dispersion in the system employed is very minimal and the liquid flow within the bed was close to plug flow, which mimics a packed bed chromatography system. Evaluation on the effect of flow velocities and bed height on the performance of Streamline DEAE using feedstock containing heat-treated crude Escherichia coli homogenate of different biomass concentrations was carried out in this study. The advantages and disadvantages as well as the problems encountered during recovery of $\mathrm{HBcAg}$ with aforementioned parameters are also discussed in this paper.
\end{abstract}

Keyword: EBAC; Liquid velocity; Bed expansion; RTD; Feedstock 